\title{
La consolidación de la política de cambio climático en la agenda de
}

la República de India ${ }^{1}$

Aldana Belén Fouquet ${ }^{2}$

\section{Contribución en la Sección Debates Internacionales}

Resumen: En el siglo XXI, la problemática del cambio climático se presenta como uno de los retos más desafiantes para la comunidad internacional. Por una parte, una de sus mayores causas es el gran incremento de la concentración de gases de efecto invernadero (GEls) producto de emisiones generadas por los países más desarrollados y las potencias emergentes. Por otro lado, sus principales manifestaciones se han observado en consecuencias devastadoras en el ambiente durante el último tiempo. En India, el abordaje de esta temática fue evolucionando de acuerdo a las condiciones del contexto político interno, el cual en los años posteriores a la independencia estuvo caracterizado por el tratamiento de cuestiones estructurales en lo que hicieron a la conformación de Estado-nación, no permitiendo una gran profundización del debate respecto de las cuestiones ambientales. No obstante, la llegada al gobierno de Manmohan Singh en el año 2004, marcó el inicio de un punto de inflexión en el tratamiento, proceso que fue retomado posteriormente con la asunción de Narendra Modi en 2014, líder del Bharatiya Janata Party. En este sentido, el presente artículo se propone abordar la consolidación de la política de cambio climático de la República de India contrastando las propuestas realizadas en la materia por el país asiático tanto a nivel interno como externo desde 2004 a 2018.

\section{Introducción}

La Convención Marco de las Naciones Unidas sobre Cambio Climático (CMNUCC), adoptada en 1992 en la Cumbre de Río, define al cambio climático como "un cambio de clima atribuido directa o indirectamente a la actividad humana que altera la composición de la atmósfera mundial y que se suma a la variabilidad natural del clima observada durante períodos de tiempo comparables" (CMNUCC, 1992, p. 6). Por lo tanto, a partir de esta

\footnotetext{
${ }^{1}$ Este trabajo se enmarca en los resultados de una investigación mayor correspondiente a una tesina de grado de carácter obligatorio para la obtención del título de Licenciada en Relaciones Internacionales otorgado por la Facultad de Ciencia Política y Relaciones Internacionales de la Universidad Nacional de Rosario (UNR, Argentina).

2 Licenciada en Relaciones Internacionales por la Universidad Nacional de Rosario (UNR, Argentina). Miembro graduada del Grupo de Estudios sobre India y el Sudeste Asiático de Rosario (GEIRSA), perteneciente al Programa de Relaciones y Cooperación Sur-Sur (PRECSUR, UNR).

Correo electrónico: aldana.fouquet@gmail.com
} 
conceptualización, se deduce que esta problemática ha ocupado un lugar cada vez mayor en los espacios de negociación multilaterales.

En lo que respecta a nuestro caso de estudio, India está geográfica y ecológicamente expuesta a grandes riesgos tales como inundaciones, sequías, calor extremo, entre otros, siendo uno de los países más vulnerables a los impactos de las variaciones del clima (Hakala, 2019). Por estas razones, y considerando que está posicionado entre los cinco principales emisores globales de GEls, el país se encuentra bajo una gran presión para mantener hábilmente un camino de desarrollo sostenible y aprovechar sus recursos de manera eficiente (Government of India, 2012). Sin embargo, durante el período de Guerra Fría, e inicios de la Posguerra Fría, las problemáticas ambientales, y en particular la cuestión relativa al cambio climático, no se vislumbraron como tópicos de agenda con un papel relevante y demasiado protagónico para los funcionarios/as indios/as.

Bajo los considerandos expresados hasta aquí, el presente artículo tiene como objeto estudiar la consolidación de la política de cambio climático de la República de India desde una perspectiva interméstica, contrastando las propuestas realizadas en la materia por el país asiático tanto a nivel doméstico como en el plano internacional durante el período 2004-2018.

\section{El tratamiento de la problemática del cambio climático en India (2004-2018)}

Hacia el año 2004, Manmohan Singh, miembro del partido del Congreso Nacional Indio, ocupó el cargo de Primer Ministro por dos períodos consecutivos, con lo cual su gobierno se extendió hasta 2014. Para aquel entonces, India experimentó la continuación del programa de liberalización económica iniciado durante el gobierno de Narasimha Rao (19911997) y comenzó a ser considerada como poder medio emergente ${ }^{3}$. Asimismo, India presentó un desempeño macroeconómico sostenido y los índices históricos de ascenso de la economía alimentaron la demanda de energía, en un contexto de rápida urbanización e industrialización, aumento de los ingresos y crecimiento del uso de productos intensivos en energía (Dussort, 2013, p. 50). Es por lo dicho que, inevitablemente, observamos un incremento en los niveles de contaminación ambiental, lo cual posicionó a India como uno de los países más contaminantes del mundo.

Ahora bien, si nos referimos puntualmente al abordaje de la temática del cambio climático durante el gobierno de Singh, se observa que India vivenció un cambio en la forma de considerar las cuestiones ambientales. En tal sentido, esta modificación se plasmó en una

\footnotetext{
${ }^{3}$ El concepto hace referencia a un "Estado que sobre la base de significativas capacidades materiales desarrolla un creciente activismo internacional en ámbitos multilaterales, tanto en su entorno regional más próximo, como en sucesivas y simultáneas negociaciones globales. Asimismo, se autopercibe y es percibido por otros agentes como diferente tanto de las restantes unidades estatales (pequeñas o iguales), como de las grandes potencias tradicionales" (Giaccaglia, 2017, p. 433).
} 
mayor particularización sobre la preocupación de la degradación planetaria, abordando al tema con mayor interés y responsabilidad tanto a nivel interno como externo, principalmente desde el año 2008.

Primeramente, debemos dar a conocer que se llevaron adelante diversas iniciativas a nivel doméstico antes de arribar a 2008, el cual se plantea como punto de quiebre en el presente trabajo. Por mencionar algunas políticas gubernamentales, en 2005 se constituyó el Grupo Consultivo sobre Cambio Climático y al año siguiente (2006), se diagnosticó que el aumento de las emisiones vehiculares era una de las principales causas del deterioro de la calidad del ambiente urbano. Todas estas decisiones nacionales, fueron conduciendo a un abordaje más comprehensivo de la problemática ambiental, de manera que en el año 2006 se creó el Consejo del Primer Ministro sobre Cambio Climático (PMCCC por sus siglas en inglés) con el propósito de desarrollar una respuesta coordinada a nivel interno (Venkatshamy y George, 2012, p. 301).

Hacia el año 2007 el Panel Intergubernamental de Cambio Climático (IPCC por sus siglas en inglés), organismo de referencia global en lo que respecta a la ciencia climática, publicó su cuarto informe de evaluación e instó a las naciones a intensificar sus responsabilidades para detener el aumento de la temperatura a nivel global. En este marco, el Consejo del Primer Ministro sobre Cambio Climático lanzó el Plan de Acción Nacional de Cambio Climático (NAPCC por sus siglas en inglés) en el año 2008, iniciativa clave y sumamente relevante a nivel local. Este plan vinculó la seguridad energética y la acción climática a través del "enfoque de co-beneficio", concepto que hace referencia a los efectos positivos que una política o medida dirigida a un objetivo en particular, podrían tener sobre otros objetivos, independientemente del efecto neto sobre el bienestar social general (Mayrhofer y Gupta, 2016, p. 22).

Teniendo en cuenta los avances que suscitó la temática del cambio climático a nivel internacional, la Comisión de Planificación india, por iniciativa del Ministerio de Medioambiente y Bosques, reconoció al cambio climático como un área sumamente importante donde era necesaria una intervención activa. En tal sentido, observamos una nueva forma de abordar la problemática ya que, desde 2011, los informes anuales del mencionado Ministerio comenzaron a incluirla en un capítulo particular, analizado de manera conjunta con la cuestión del desarrollo sostenible (Government of India, 2011). Por lo tanto, consideramos que lo dicho es de relevancia ya que, en los informes de los años previos, solamente se realizaba un breve abordaje en un pequeño subapartado.

Ahora bien, hacia 2014, Narendra Modi, miembro del Bharatiya Janata Party (BJP), se convirtió en el nuevo Primer Ministro de India, obteniendo una mayoría absoluta inédita en los últimos treinta años del país asiático y prescindiendo del apoyo de otros partidos políticos 
(De Pedro, 2015). Con la nueva administración, la preocupación por el cambio climático en India fue en ascenso y desde 2014 hubo dos cambios fundamentales al interior del Ministerio de Medioambiente y Bosques, los cuales mostraron la gran preeminencia del tema en la agenda gubernamental del BJP. En primer lugar, en el año 2014, se creó una División sobre Cambio Climático en dicho Ministerio, que se encargó de lograr una mejora en los impactos ambientales mediante el incremento de políticas y acciones nacionales apropiadas. En segundo lugar, Narendra Modi modificó la nomenclatura del mencionado organismo, ya que pasó a ser denominado Ministerio de Medioambiente, Bosques y Cambio Climático ${ }^{4}$.

Asimismo, se idearon numerosos proyectos a nivel local, siendo ejemplos de los mismos la iniciativa Smart Cities, Clean India, o la implementación de combustibles alternativos a base de hidrógeno para ayudar a combatir la contaminación y mejorar la calidad de las emisiones (Ellis Peterse, 2019).

En cuanto al posicionamiento y propuestas llevadas adelante en el ámbito internacional durante el período seleccionado para esta investigación, se observa que India intentó demostrar constantemente una mayor visibilidad e intensificación en el plano de los compromisos internacionales ya desde inicios del mandato de Singh. Nueva Delhi ratificó y formó parte del Protocolo de Kioto a la CMNUCC, el cual entró en vigor en 2005 constituyendo un primer intento de implementación de la Convención; firmó en 2009 el estatuto de la Agencia Internacional de Energías Renovables (IRENA en inglés) convirtiéndose en el miembro fundador número 77 , entre otras participaciones destacables.

En función de lo expresado, podemos afirmar que hubo una clara continuidad del comportamiento en el escenario internacional durante el gobierno de Modi en virtud de hacer oír las consideraciones del país respecto del cambio climático. En este sentido, el BJP desarrolló una participación sumamente activa enviando delegaciones indias a distintos foros que trataron la temática estudiada, tales como la Conferencia de las Partes, el grupo BASIC, el G20 o el Foro BRICS. Asimismo, India conformó una iniciativa a nivel global junto con Francia denominada Alianza Solar Internacional, un proyecto lanzado en 2015 e inaugurado en el año 2018. Tal Alianza se ideó con el objetivo primordial de transferir tecnología y financiar proyectos de energía solar en países en vías de desarrollo, generando una capacidad de 1.000 gigavatios de este tipo de energía hasta el año 2030 e incorporando a 121 naciones (Alares, 2018).

En este contexto y en virtud del imperativo global por mantener el aumento de la temperatura media mundial muy por debajo de los $2^{\circ} \mathrm{C}$, haciendo esfuerzos por limitarla en $1.5^{\circ} \mathrm{C}$, el nuevo gobierno también se vio comprometido en las negociaciones del Acuerdo de

\footnotetext{
${ }^{4}$ Información extraída de: https://cutt.ly/uWx98ii

(Consulta: 15 de enero de 2020).
} 
París en 2015, participando de la Conferencia de las Naciones Unidas sobre el Cambio Climático (COP) 21 y ratificando el nuevo documento global rápidamente, a pesar de haber intentado paralizar las negociaciones cuando se alejaban de las ideas centrales que hacen al interés nacional.

India, como uno de los países líderes del mundo en desarrollo, en todo momento llevó adelante el principio de responsabilidades comunes pero diferenciadas (CBDR por sus siglas en inglés), concepto clave que caracteriza su posicionamiento en las mesas de debate internacional y que hace alusión a que los países desarrollados tienen mayores responsabilidades ambientales que los países en desarrollo. La causa subyace en los patrones de consumo y de producción históricos mantenidos por los primeros desde comienzos de la Revolución Industrial. Nueva Delhi, más allá de sostener la defensa de sus intereses nacionales, finalmente dio el visto bueno para arribar a un nuevo acuerdo climático. Este pragmatismo, supuso que por más que India quedó inevitablemente relacionada en el tratamiento de uno de los puntos más conflictivos para lograr un consenso en 2015, firmó el documento manifestando su adhesión a las nuevas condiciones del acuerdo global.

No obstante, más allá de que India se esforzó por no quedar atrás en los debates climáticos internacionales y en desarrollar políticas gubernamentales a nivel interno que sean amigables con el ambiente, el país aumentó sus emisiones en un 7.2\% en 2018 de acuerdo a los niveles de 2017 (Troillet et al., 2019) y que sigue formando parte de los cinco principales emisores mundiales. Por lo tanto, los compromisos deben continuar reforzándose en función de obtener efectos más consistentes a nivel doméstico que vayan en sintonía con los diagnósticos y las recomendaciones realizadas por la ciencia climática.

\section{A modo de cierre}

Retomando las consideraciones anteriores, se puede concluir que desde inicios del siglo XXI India comenzó a manifestar un mayor interés respecto del abordaje del cambio climático tanto a nivel interno como externo, siendo incluida la temática en la agenda gubernamental de una manera mucho más activa desde el gobierno de Singh lo que posibilita afirmar la existencia de una retroalimentación constante entre ambos espacios. De esta forma, desde el año 2004, pero más particularmente desde 2008, se esbozó una política de cambio climático, sustentada en múltiples iniciativas que tuvieron como objetivo central reducir las emisiones de GEls. Posteriormente, al arribar al primer gobierno de Narendra Modi, se observaron más continuidades que retrocesos en el tratamiento del tema, pudiendo aseverar que el cambio de color político supuso una evolución constante en la presentación de propuestas ambientales tanto a nivel doméstico como a nivel internacional. 


\section{Referencias}

Alares, D. A. (11 de marzo de 2018). La Alianza Solar arranca en la India con 1.400 millones de dólares en ayudas. Agencia EFE.

https://www.efe.com/efe/america/economia/la-alianza-solar-arranca-en-india-con1-400-millones-de-dolares-ayudas/20000011-3549110

Convención Marco de las Naciones Unidas sobre el Cambio Climático (1992). Disponible en https://unfccc.int/files/essential_background/background_publications_htmlpdf/ap plication/pdf/convsp.pdf

De Pedro, N. (2015). Un año de Narendra Modi: India y el mundo siguen expectantes. Notes Internacionals CIDOB, 120, junio. https://cutt.ly/WWx0ab9

Dussort, M. N. (2013), ¿Por qué India pasó a ser un consumidor energético global?: Un análisis sobre el contexto energético interno del gigante asiático. Revista NEIBA, Cuadernos Argentina-Brasil, 2(1), 47-60.

Ellis Petersen, H. (13 de noviembre de 2019). India says it plans to use hydrogen-based fuel to tackle air crisis. The Guardian. https://cutt.ly/VWx0xqB

Fouquet, A. (2020). La política de cambio climático de la República de India en el marco de la diplomacia de cumbres. Los casos de la COP, el G20 y el Foro BRICS en el período 2008-2018. Tesina de grado, Facultad de Ciencia Política y Relaciones Internacionales, Universidad Nacional de Rosario. Disponible en: https://cutt.ly/oWx0neD

Giaccaglia, C. (2017). Poderes tradicionales, emergentes y re-emergentes: relaciones ambiguas pero pragmáticas. Foro internacional, 57(2), 422-459. https://doi.org/10.24201/fi.v57i2.2364

Government of India. Ministry of Environment and Forest (2011). Annual Report 2010-2011. New Delhi.

Government of India. Ministry of Environment and Forest (2012). Annual Report 2011-2012. New Delhi.

Hakala, E. S. (2019). Global responsibility as strategic interest. India and the geoeconomics of climate change. FIIA Working Paper, Vol. 104, August 2018. Finnish Institute of International Affairs.

Mayrhofer, J. y Gupta, J. (2016). The science and politics of co benefits in climate policy.

Environmental Science \& Policy, 57, 22-30.

https://doi.org/10.1016/j.envsci.2015.11.005 
Triollet, R., Mccafferty, E. J., Alvarez Martinez, A. F., Tóth, B., Bellan, E. y Al Khudhairy, D. (2019). JRC Annual Report 2019, Publications Office of the European Union. Luxembourg. DOI 10.2760/546288.

https://publications.jrc.ec.europa.eu/repository/handle/JRC119146

Venkatshamy, K y George, P. (Eds.) (2012). Grand strategy for India: 2020 and beyond. Pentagon Security International. 